\title{
REDUCING HUGE GYROSCOPIC EIGENPROBLEMS BY AUTOMATED MULTI-LEVEL SUBSTRUCTURING
}

\author{
KOLJA ELSSEL AND HEINRICH VOSS *
}

\begin{abstract}
Simulating numerically the sound radiation of a rolling tire requires the solution of a very large and sparse gyroscopic eigenvalue problem. Taking advantage of the automated multilevel substructuring (AMLS) method it can be projected to a much smaller gyroscopic problem, the solution of which however is still quite costly since the eigenmodes are non-real and complex arithmetic is necessary. This paper discusses the application of AMLS to huge gyroscopic problems and the numerical solution of the AMLS reduction. A numerical example demonstrates the efficiency of AMLS.
\end{abstract}

Key words. Eigenvalue, AMLS, gyroscopic eigenproblem, substructuring, nonlinear eigenproblem, minmax characterization

AMS subject classification. $65 \mathrm{~F} 15,65 \mathrm{H} 50$

1. Introduction. A great deal of the overall sound source of road traffic is caused by rolling noise of road vehicles. For passenger cars at speeds above $40 \mathrm{~km} / \mathrm{h}$, and for trucks above $60 \mathrm{~km} / \mathrm{h}$ the major source of traffic noise is due to the sound radiation of rolling tires (cf. [19, 21]). According to Nackenhorst and von Estorff [21] the simulation of the tire noise is performed in three steps. First, the nonlinear tire deflections under steady state conditions are computed using an Arbitrary Lagrangian Eulerian (ALE) approach. Next, the transient vibrations governed by the eigenpairs of a gyroscopic eigenvalue problem

$$
Q(\omega) x:=K x+\omega G x+\omega^{2} M x=0 .
$$

are assumed to be superimposed onto the nonlinear deflections. Finally, the acoustic analysis is carried out solving Helmholtz's equation where the normal velocities at the wheel surface, extracted from the vibration analysis, are taken as boundary conditions.

In this paper we consider only the second step, i.e. the numerical solution of the eigenproblem (1.1) where $K$ is the stiffness matrix modified by the presence of centripetal forces, $M$ is the mass matrix, and $G$ is the gyroscopic matrix stemming from the Coriolis force. Clearly, $K$ and $M$ are symmetric and positive definite, and $G$ is skew-symmetric.

Due to the complicated interior structure of a belted tire the matrices $K, M$ and $G$ of a sufficiently accurate FE model are very large and sparse. Moreover, for the acoustic analysis many eigenpairs not necessarily at the end of the spectrum are needed. Therefore, well-established sparse eigensolvers of Arnoldi type with shift and invert techniques [15] for a linearization of problem (1.1), methods which are based on structure preserving linearizations like SHIRA [18], and iterative projection methods for nonlinear eigenproblems [17] are very costly since LU factorizations of complex valued matrices $Q\left(\omega_{j}\right)$ for several parameters $\omega_{j}$ are required.

Over the last few years, a new method for huge eigenvalue problems, known as Automated Multi-Level Substructuring (AMLS), has been developed by Bennighof and co-authors, and has been applied to frequency response analysis of complex structures $[2,3,11]$. Here the large finite element model is recursively divided into very many

*Institute of Numerical Simulation, Hamburg University of Technology, D-21071 Hamburg, Germany (\{elssel, voss\}@tu-harburg.de) 
substructures on several levels based on the sparsity structure of the system matrices. Assuming that the interior degrees of freedom of substructures depend quasistatically on the interface degrees of freedom, and modeling the deviation from quasistatic dependence in terms of a small number of selected substructure eigenmodes, the size of the finite element model is reduced substantially yet yielding satisfactory accuracy over a wide frequency range of interest. Recent studies ([11, 13, 24], e.g.) in vibroacoustic analysis of passenger car bodies where huge $\mathrm{FE}$ models with more than six million degrees of freedom appear and several hundreds of eigenfrequencies and eigenmodes are needed have shown that AMLS is considerably faster than Lanczos type approaches for this sort of problems.

From a mathematical point of view, AMLS is nothing else but a projection method where the large problem under consideration is projected to a subspace spanned by a small number of eigenmodes of undamped clamped substructures on several levels. With respect to this basis the projection of the stiffness matrix $K$ becomes diagonal, and the mass matrix $M$ is projected to a generalized arrowhead form (cf. Fig. 1).

An obvious way to apply AMLS to gyroscopic problems is to reduce the linear pencil $(K, M)$ by AMLS and to perform all transformations in the course of the AMLS method for the gyroscopic matrix $G$ simultaneously. Thus one arrives at a projected problem

$$
\mathcal{K} y+\omega \mathcal{G} y+\omega^{2} \mathcal{M} y=0 .
$$

Then the projection preserves the gyroscopic structure of problem (1.1), and problem (1.2) can be solved by (structure preserving) linearization [16, 18, 25] or by an iterative projection method of Arnoldi [26] or Jacobi-Davidson type [4].

Notice however, that for a realistic model of a rolling tire the projected problem can still be very large, and solving (1.2) numerically can be very costly, in particular since a large number of eigenpairs is required in the acoustic analysis, and since the eigenvectors are non-real, and solving (1.2) requires complex arithmetic.

Since the influence of the skew-symmetric matrix $\mathcal{G}$ on the eigenvectors of problem (1.2) is small compared to the matrices $\mathcal{K}$ and $\mathcal{M}$ (although not negligible), we suggest to approximate problem (1.2) further projecting it to a subspace spanned by eigenvectors of the linear symmetric eigenvalue problem

$$
\mathcal{K} z=\lambda^{2} \mathcal{M} z
$$

corresponding to eigenvalues not exceeding a given bound. These eigenvectors can be determined in real arithmetic by a well-established sparse eigensolver like ARPACK [15], and the time for solving problem (1.2) can be reduced considerably.

Our presentation is organized as follows. Section 2 summarizes the AMLS method for linear eigenvalue problems. In Section 3 we apply it to gyroscopic problems, and discuss the numerical solution of the projected gyroscopic eigenproblem. Section 4 demonstrates the efficiency of the approach by a numerical example. Although its dimension is only ca. 125.000 which does not seem to be extraordinary large we call the problem huge since the structure of its system matrices is complicated and an LU factorization of $Q(\omega)$ requires such an amount of storage and computing time that the common large eigensolvers of Arnoldi or Jacobi-Davidson type become inefficient.

2. AMLS for linear eigenproblems. In this section we summarize the Automated Multi-Level Substructuring (AMLS) method for the linear eigenvalue problem

$$
K x=\lambda^{2} M x
$$




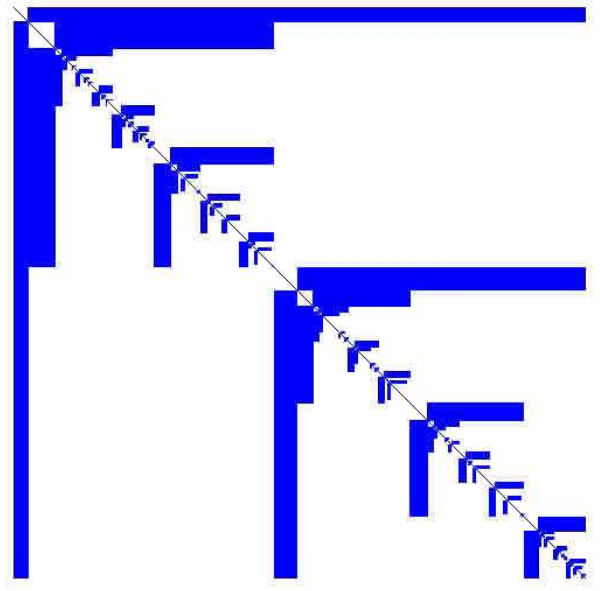

Fig. 1: Projected mass matrix

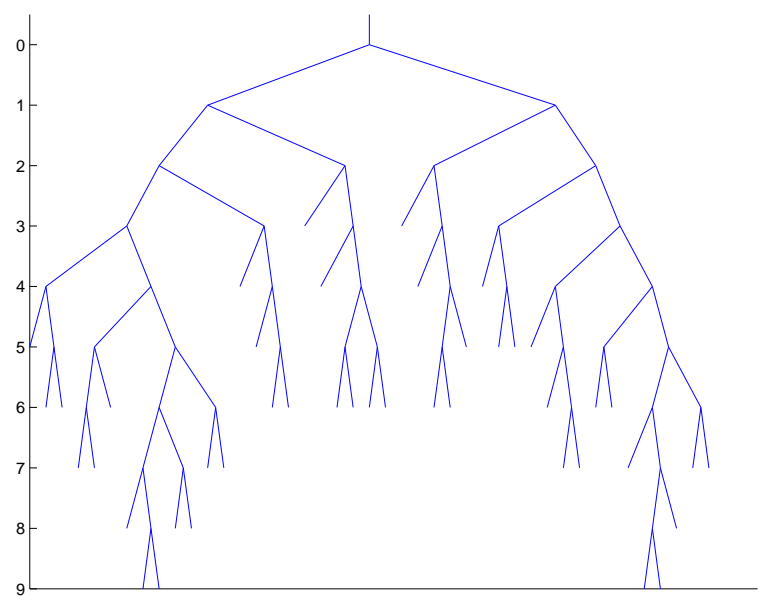

Fig. 2: Tree of a multi-level substructuring

which was developed by Bennighof and co-workers over the last few years [2, 3, 11], who applied it to solve frequency response problems involving large and complex models. Here, $K$ is the stiffness matrix and $M$ the mass matrix of a finite element model of a structure.

Similarly as in the component mode synthesis (CMS) the structure is partitioned into a small number of substructures based on the sparsity pattern of the system matrices, but more generally than in CMS these substructures in turn are substructured on a number of levels yielding a tree topology for the substructures. Fig. 2 shows an example were each substructure has at most two children.

We stress the fact that substructuring does not necessarily mean that it is obtained by a domain decomposition of a real structure, but it is understood in a purely algebraic sense. Although substructuring by hand may yield much smaller interfaces than the ones obtained by automatic partitioners (cf. [7]), the dissection of the matrices can be derived by applying a graph partitioner like CHACO [9] or METIS [12] to the undirected graph corresponding to the nonzero pattern of the matrices under consideration. These programs have been designed to construct fill-reducing orderings 
of sparse matrices for efficient factorization but have shown to be a beneficial basis of substructuring methods as well. In either case, because of its pictographic nomenclature we will use terms like substructure or eigenmode from frequency response problems when describing the AMLS method.

Substructures on the lowest level consist of a small number of degrees of freedom, which are partitioned into two sets: interface degrees of freedom which are shared with an adjacent substructure, and interior or local degrees of freedom which are only connected to degrees of freedom in their own substructure. To demonstrate the AMLS method we distinguish only between two substructures on the lowest level (corresponding to index sets $I_{1}$ and $I_{2}$ ), the interface between these substructures (with index set $I_{3}$ ) and the remaining degrees of freedom (index set $I_{4}$ ). Partitioning the displacement vector $x$ correspondingly and reordering the matrices $K$ and $M$ the eigenvalue problem (2.1) obtains the following form

$$
\left(\begin{array}{cccc}
K_{11} & O & K_{13} & K_{14} \\
O & K_{22} & K_{23} & K_{24} \\
K_{13}^{T} & K_{23}^{T} & K_{33} & K_{34} \\
K_{14}^{T} & K_{24}^{T} & K_{34}^{T} & K_{44}
\end{array}\right)\left(\begin{array}{l}
x_{1} \\
x_{2} \\
x_{3} \\
x_{4}
\end{array}\right)=\lambda\left(\begin{array}{cccc}
M_{11} & O & M_{13} & M_{14} \\
O & M_{22} & M_{23} & M_{24} \\
M_{13}^{T} & M_{23}^{T} & M_{33} & M_{34} \\
M_{14}^{T} & M_{24}^{T} & M_{34}^{T} & M_{44}
\end{array}\right)\left(\begin{array}{l}
x_{1} \\
x_{2} \\
x_{3} \\
x_{4}
\end{array}\right)
$$

To remove the off-diagonal blocks $K_{i j}, i=1,2, j=3,4$ in $K$ we apply the congruence transformation with

$$
\begin{gathered}
T^{T} K T y=\lambda T^{T} M T y, \quad y=T^{-1} x \\
T=\left(\begin{array}{cccc}
I & O & -K_{11}^{-1} K_{13} & -K_{11}^{-1} K_{14} \\
O & I & -K_{22}^{-1} K_{23} & -K_{22}^{-1} K_{24} \\
O & O & I & O \\
O & O & O & I
\end{array}\right) .
\end{gathered}
$$

Then problem (2.1) is equivalent to

$$
\left(\begin{array}{cccc}
K_{11} & O & O & O \\
O & K_{22} & O & O \\
O & O & \tilde{K}_{33} & \tilde{K}_{34} \\
O & O & \tilde{K}_{34}^{T} & \tilde{K}_{44}
\end{array}\right)\left(\begin{array}{l}
y_{1} \\
y_{2} \\
y_{3} \\
y_{4}
\end{array}\right)=\lambda\left(\begin{array}{cccc}
M_{11} & O & \tilde{M}_{13} & \tilde{M}_{14} \\
O & M_{22} & \tilde{M}_{23} & \tilde{M}_{24} \\
\tilde{M}_{13}^{T} & \tilde{M}_{23}^{T} & \tilde{M}_{33} & \tilde{M}_{34} \\
\tilde{M}_{14}^{T} & \tilde{M}_{24}^{T} & \tilde{M}_{34}^{T} & \tilde{M}_{44}
\end{array}\right)\left(\begin{array}{l}
y_{1} \\
y_{2} \\
y_{3} \\
y_{4}
\end{array}\right)
$$

where

$$
\begin{aligned}
\tilde{K}_{j j} & =K_{j j}-\sum_{i=1,2} K_{i j}^{T} K_{i i}^{-1} K_{i j}, j=3,4 \\
\tilde{K}_{34} & =K_{34}-\sum_{i=1,2} K_{i 3}^{T} K_{i i}^{-1} K_{i 4} \\
\tilde{M}_{i j} & =M_{i j}-M_{i i} K_{i i}^{-1} K_{i j}, i=1,2, j=3,4 \\
\tilde{M}_{j j} & =M_{j j}-\sum_{i=1,2}\left(M_{i j}^{T} K_{i i}^{-1} K_{i j}+K_{i j}^{T} K_{i i}^{-1} M_{i j}-K_{i j}^{T} K_{i i}^{-1} M_{i i} K_{i i}^{-1} K_{i j}\right), j=3,4 \\
\tilde{M}_{34} & =M_{34}-\sum_{i=1,2}\left(M_{i 3}^{T} K_{i i}^{-1} K_{i 4}+K_{i 3}^{T} K_{i i}^{-1} M_{i 4}-K_{i 3}^{T} K_{i i}^{-1} M_{i i} K_{i i}^{-1} K_{i 4}\right) .
\end{aligned}
$$

Taking advantage of the modal representation

$$
K_{j j} \Phi_{j}=M_{j j} \Phi_{j} \Omega_{j}, \quad \Phi_{j}^{T} M_{j j} \Phi_{j}=I, \quad j=1,2,
$$


of the substructures under consideration, and applying the further congruence transformation by the block diagonal matrix $\tilde{T}=\operatorname{diag}\left\{\Phi_{1}, \Phi_{2}, I, I\right\}$ we obtain the equivalent eigenvalue problem

$$
\left(\begin{array}{cccc}
\Omega_{1} & O & O & O \\
O & \Omega_{2} & O & O \\
O & O & \tilde{K}_{33} & \tilde{K}_{34} \\
O & O & \tilde{K}_{34}^{T} & \tilde{K}_{44}
\end{array}\right)\left(\begin{array}{c}
z_{1} \\
z_{2} \\
z_{3} \\
z_{4}
\end{array}\right)=\lambda\left(\begin{array}{cccc}
I & O & \hat{M}_{13} & \hat{M}_{14} \\
O & I & \hat{M}_{23} & \hat{M}_{24} \\
\hat{M}_{13}^{T} & \hat{M}_{23}^{T} & \tilde{M}_{33} & \tilde{M}_{34} \\
\hat{M}_{14}^{T} & \hat{M}_{24}^{T} & \tilde{M}_{34}^{T} & \tilde{M}_{44}
\end{array}\right)\left(\begin{array}{c}
z_{1} \\
z_{2} \\
z_{3} \\
z_{4}
\end{array}\right)
$$

where

$$
\hat{M}_{i j}=\Phi_{i}^{T} \tilde{M}_{i j}, i=1,2, j=3,4 .
$$

These transformations are applied to all substructures on the finest level. The resulting eigenproblem is a quasistatic-modal representation of the structure which is known as Craig-Bampton form in component mode synthesis method.

It is well known that the high frequency modes of the substructures do not influence the low frequency modes of the entire structure very much. Hence, similarly as in the component mode synthesis method we can reduce the dimension of the eigenvalue problem (2.6) considerably if we delete rows and columns which correspond to frequencies of the substructures exceeding a given cut-off frequency.

The resulting eigenproblem is the projection of problem (2.1) to the subspace spanned by all interface degrees of freedom and the kept modes of the substructures of the finest substructuring. It obtains the same block form as problem (2.6), although the two leading block rows and columns are of much smaller dimension:

$$
\left(\begin{array}{cccc}
\Omega_{1}^{\prime} & O & O & O \\
O & \Omega_{2}^{\prime} & O & O \\
O & O & \tilde{K}_{33} & \tilde{K}_{34} \\
O & O & \tilde{K}_{34}^{T} & \tilde{K}_{44}
\end{array}\right)\left(\begin{array}{c}
z_{1}^{\prime} \\
z_{2}^{\prime} \\
z_{3} \\
z_{4}
\end{array}\right)=\lambda\left(\begin{array}{cccc}
I & O & \hat{M}_{13}^{\prime} & \hat{M}_{14}^{\prime} \\
O & I & \hat{M}_{23}^{\prime} & \hat{M}_{24}^{\prime} \\
\hat{M}_{13}^{\prime T} & \hat{M}_{23}^{\prime T} & \tilde{M}_{33} & \tilde{M}_{34} \\
\hat{M}_{14}^{\prime T} & \hat{M}_{24}^{\prime T} & \tilde{M}_{34}^{T} & \tilde{M}_{44}
\end{array}\right)\left(\begin{array}{c}
z_{1}^{\prime} \\
z_{2}^{\prime} \\
z_{3} \\
z_{4}
\end{array}\right),
$$

Once substructures on the lowest level have been transformed and reduced by modal projection they are assembled to parent substructures on the next level. Again interface and local degrees of freedom are identified, and the substructure models are transformed similarly as on the lowest level.

For instance, for the parent substructure consisting of the three leading block rows and columns of the modal reduction (2.7) of problem (2.6) we remove the off-diagonal block $\tilde{K}_{34}$ by a congruence transformation similar to (2.3) yielding

$$
\left(\begin{array}{cccc}
\Omega_{1}^{\prime} & O & O & O \\
O & \Omega_{2}^{\prime} & O & O \\
O & O & \tilde{K}_{33} & O \\
O & O & O & \hat{K}_{44}
\end{array}\right)\left(\begin{array}{c}
z_{1}^{\prime} \\
z_{2}^{\prime} \\
z_{3}^{\prime} \\
z_{4}^{\prime}
\end{array}\right)=\lambda\left(\begin{array}{cccc}
I & O & \hat{M}_{13}^{\prime} & \bar{M}_{14} \\
O & I & \hat{M}_{23}^{\prime} & \bar{M}_{24} \\
\hat{M}_{13}^{\prime T} & \hat{M}_{23}^{\prime T} & \tilde{M}_{33} & \bar{M}_{34} \\
\bar{M}_{14}^{T} & \bar{M}_{24}^{T} & \bar{M}_{34}^{T} & \bar{M}_{44}
\end{array}\right)\left(\begin{array}{c}
z_{1}^{\prime} \\
z_{2}^{\prime} \\
z_{3}^{\prime} \\
z_{4}^{\prime}
\end{array}\right)
$$

with

$$
\begin{aligned}
\bar{M}_{i 4} & =\hat{M}_{i 4}^{\prime}-\hat{M}_{i 3}^{\prime} \tilde{K}_{33}^{-1} \tilde{K}_{34}, i=1,2,3 \\
\bar{M}_{44} & =\tilde{M}_{44}-\tilde{M}_{34}^{T} \tilde{K}_{33}^{-1} \tilde{K}_{34}-\tilde{K}_{34}^{T} \tilde{K}_{33}^{-1} \tilde{M}_{34}+\tilde{K}_{34}^{T} \tilde{K}_{33}^{-1} \tilde{M}_{33} \tilde{K}_{33}^{-1} \tilde{K}_{34} .
\end{aligned}
$$


To perform the modal reduction of the interior degrees of freedom of the current substructure one would have to solve the eigenvalue problem

$$
\left(\begin{array}{ccc}
\Omega_{1}^{\prime} & O & O \\
O & \Omega_{2}^{\prime} & O \\
O & O & \tilde{K}_{33}
\end{array}\right)\left(\begin{array}{l}
w_{1} \\
w_{2} \\
w_{3}
\end{array}\right)=\omega\left(\begin{array}{ccc}
I & O & \hat{M}_{13}^{\prime} \\
O & I & \hat{M}_{23}^{\prime} \\
\hat{M}_{13}^{\prime T} & \hat{M}_{23}^{\prime T} & \tilde{M}_{33}
\end{array}\right)\left(\begin{array}{l}
w_{1} \\
w_{2} \\
w_{3}
\end{array}\right) .
$$

However, since the number of interior degrees of freedom of substructures grows too large in the course of the algorithm, we reduce the dimension only taking advantage of the eigenvalue problem corresponding to the right lower diagonal block, i.e.

$$
\tilde{K}_{33} \Phi_{3}=\tilde{M}_{33} \Phi_{3} \Omega_{3}, \Phi_{3}^{T} \tilde{M}_{33} \Phi_{3}=I .
$$

Applying the congruence transformation with $T=\operatorname{diag}\left\{I, I, \Phi_{3}, I\right\}$ to problem (2.6), and dropping all rows and columns in the third block if the corresponding eigenvalue exceeds the cut-off frequency we further reduce the dimension of the eigenproblem.

Assembly to higher-level substructures, transformation to quasistatic-modal representation, and projection to subspaces spanned by modes of diagonal blocks not exceeding the cut-off frequency continues, until we arrive at an approximate model for the entire structure. We end up with a projected eigenproblem

$$
\mathcal{K} y=\lambda \mathcal{M} y,
$$

where the stiffness matrix $\mathcal{K}$ has become diagonal, and the mass matrix is projected to a matrix $\mathcal{M}$ the diagonal of which is the identity, and the only off-diagonal blocks containing non-zero elements are the ones describing the coupling of the substructures and its interfaces. Fig. 1 shows the generalized arrowhead structure of the resulting mass matrix.

Once the multi-level substructuring transformation of the problem (2.1) has been accomplished, the substructure modes can be collected in a matrix $\Phi_{\text {AMLS }}$, and if $(\lambda, y)$ is an eigenpair of the projected problem $(2.11)$ then $(\lambda, x)=\left(\lambda, \Phi_{\text {AMLS }} y\right)$ is an approximate eigenpair of problem (2.1) called Ritz-pair.

The cost of performing the projection above consists of the cost of obtaining the submatrices $\Phi_{j}$ of $\Phi_{\mathrm{AMLS}}$, and transforming the substructure stiffness and mass matrices $K$ and $M$. Notice that for every substructure only a partial eigenproblem has to be solved, and only a small number of eigenpairs is needed. Moreover, the eigenproblems are usually very small because most of the local degrees of freedom of a substructure are local degrees of the substructures of the next lower level which form the current substructure. Hence, the part of the substructure stiffness matrix corresponding to these degrees of freedom is already diagonal, and we only consider those local degrees of freedom which did not have this property on the next lower level, i.e. those interface degrees of freedom of the next lower level which are not interface degrees of freedom on the current level.

Differently from the iterative projection methods like Lanczos [14], Arnoldi [1], rational Krylov [22] or Jacobi-Davidson [23] where approximations to the wanted eigenpairs are obtained from projections of problem (2.1) to subspaces which are expanded in the course of the algorithm, AMLS is a one shot method, i.e. after having chosen a cut-off frequency or another dropping rule the method produces one fixed subspace $V$ and the corresponding projected eigenproblem. If the computed approximate eigenpairs turn out to be not accurate enough there is no way to expand 
the subspace $V$ reusing the projected problem but one has to repeat the reduction with a higher cut-off frequency.

In a recent paper Yang et al. [27] considered the component mode synthesis method, and they obtained a simple heuristic for choosing spectral components from each substructure suggesting to drop all eigenpairs $(\omega, \phi)$ of substructures in the reduction process such that

$$
\rho_{1}(\omega):=\frac{\lambda_{1}}{\omega-\lambda_{1}} \leq \tau
$$

where $\lambda_{1}$ is the smallest eigenvalue of the problem under consideration, and $\tau$ is a given tolerance.

Taking advantage of a minmax characterization of eigenvalues of nonlinear eigenproblems in [7] we proved that $\rho_{1}(\omega)$ is an a priori bound of the relative error for the smallest eigenvalue $\lambda_{1}$ of problem $(2.1)$, and that similarly $\rho_{j}(\omega):=\lambda_{j} /\left(\omega-\lambda_{j}\right)$ is an apriori bound of the relative error for higher eigenvalues $\lambda_{j}$ not exceeding $\omega$. More generally we proved the following theorem for the AMLS method:

TheOREM 2.1. Let $0<\lambda_{1} \leq \lambda_{2} \leq \cdots \leq \lambda_{n}$ be the eigenvalues of problem (2.1), and let

$$
\tilde{\lambda}_{1} \leq \tilde{\lambda}_{2} \leq \tilde{\lambda}_{m}<\min _{\nu=0, \ldots, p} \omega_{\nu} \leq \tilde{\lambda}_{m+1} \leq \ldots
$$

be the eigenvalues of the projected eigenproblem by AMLS with p levels of substructuring where on the $\nu$-th level eigenvalues exceeding $\omega_{\nu}$ are neglected. Then it holds

$$
\frac{\tilde{\lambda}_{j}-\lambda_{j}}{\lambda_{j}} \leq \prod_{\nu=0}^{p}\left(1+\frac{\tilde{\lambda}_{j}}{\omega_{\nu}-\tilde{\lambda}_{j}}\right)-1, \quad j=1, \ldots, m .
$$

Numerical examples demonstrate that these a priori bounds overestimate the real relative errors by a factor up to 100 (although an example in [7] demonstrates that the a priori bound can not be improved without further assumptions). They suggest the following rule of thump for dropping frequencies: if one is interested to obtain the eigenvalues smaller than $\hat{\omega}$ with relative errors not exceeding $1 \%$ then a cut-off frequency $10 \hat{\omega}$ usually suffices.

3. AMLS for gyroscopic eigenproblems. We consider the gyroscopic eigenproblem (1.1) in its equivalent form

$$
\tilde{Q}(\lambda) x:=K x+i \lambda G x-\lambda^{2} M x=0, \quad \lambda=i \omega,
$$

where $K$ and $M$ are symmetric and positive definite and $G$ is skew-symmetric.

Since the influence of the gyroscopic matrix $G$ on the eigenvectors is usually not very high compared to the mass and stiffness matrix, it is reasonable to neglect the linear term when defining the basis transformations and modal reductions corresponding to the substructures. Hence we apply the AMLS reduction to the pencil $(K, M)$, and we perform the same congruence transformations and modal reductions for the skew-symmetric matrix $G$. Thus we obtain the reduced model

$$
\mathcal{K} y+i \lambda \mathcal{G} y-\lambda^{2} \mathcal{M} y=0,
$$

where the stiffness and mass matrix have the same structure as in the linear case. If the sparsity pattern of $G$ matches the one of $K$ and $M$, then the gyroscopic matrix 
$\mathcal{G}$ is a skew-symmetric block matrix with generalized arrowhead form. It contains diagonal blocks corresponding to the (reduced) substructures and interfaces, and only off-diagonal blocks describing the coupling of a substructure and its interface contain non-zero elements.

Notice, that all projectors are real, and therefore the reduction can be performed in real arithmetic.

If the dimension of problem (3.2) is very small, a method at hand is to consider the linearization

$$
\left(\begin{array}{cc}
i \mathcal{G} & \mathcal{K} \\
\mathcal{K} & O
\end{array}\right)\left(\begin{array}{c}
\lambda x \\
x
\end{array}\right)=\lambda\left(\begin{array}{cc}
\mathcal{M} & O \\
O & \mathcal{K}
\end{array}\right)\left(\begin{array}{c}
\lambda x \\
x
\end{array}\right)
$$

of problem (3.2) and to apply any dense solver.

For very large gyroscopic problems (for instance a realistic model of a rolling tire) the dimension of the projected problem (3.2) will still be quite large. In this case (3.2) can be solved by an iterative projection method taking advantage of the minmax characterization of the positive eigenvalues of (3.2) [26], by a structure preserving Lanczos method [18], or by a sparse solver of the linearization (3.3) like ARPACK [15]. In all of these cases the solution requires complex arithmetic.

Since the influence of the gyroscopic part on the eigenvectors of (3.2) is not very large, problem (3.2) can often be solved even more efficiently. First it is projected to a subspace spanned by eigenvectors of the linear problem

$$
\mathcal{K} z=\lambda \mathcal{M} z
$$

corresponding to eigenvalues not exceeding a threshold $\sigma \omega_{\max }^{2}$ where $\omega_{\max }$ is an upper bound of the wanted eigenvalues of problem (1.1) and $\sigma>1$ is a small constant, and then the projected problem is solved by a dense solver.

A disadvantage of the approach above may be the fact that the eigenvectors of the clamped substructures not taking into account the gyroscopic part are not appropriate to model the deviation from quasi-static behavior of the substructures. We therefore in [8] considered a variant of the AMLS method for gyroscopic problems using the fact that the restriction

$$
K_{j j} \eta+i \lambda G_{j j} \eta-\lambda^{2} M_{j j} \eta=0
$$

of problem (3.1) to each of the substructures is gyroscopic itself, and has similar properties as the linear problem $K_{j j} \eta=\lambda^{2} M_{j j} \eta$ : its eigenvalues are real and come in pairs $\{\lambda,-\lambda\}$, its positive eigenvalues (ordered by magnitude) can be characterized as minmax values of a Rayleigh functional (cf. [6]), and the corresponding eigenvectors are linearly independent. Hence, the matrix $\Phi_{j}$ in the basis transformation (2.5) can be replaced by a matrix the columns of which are the eigenvectors of (3.5), and all columns corresponding to eigenvalues exceeding a given threshold are discarded in the projected problem. This method has indeed better approximation properties than the original AMLS method. However, the eigenvectors of (3.3) are non-real, and the reduction has to be performed in complex arithmetic from the very beginning, and numerical examples demonstrate that this additional effort does not pay [8].

4. Numerical experiments. AMLS is applied to a finite element model of a deformable wheel rolling on a rigid plane surface which is obtained by an Arbitrary Lagrangian Eulerian (ALE) formulation according to the derivation and presentation in [20]. Our model of a rotating tire consists of 39204 brick elements with 124992 


\begin{tabular}{|l|r|rr|rr|}
\hline & Preconditioner & \multicolumn{2}{|c|}{ SuperLU } & \multicolumn{2}{|c|}{ MA 57 } \\
& & memory & CPU time & memory & CPU time \\
\hline Linearization & complex & 6.04 GByte & $3910 \mathrm{sec}$. & & \\
nonlin. Arnoldi & real & 2.70 GByte & $1940 \mathrm{sec}$. & 2.86 GByte & $1080 \mathrm{sec}$. \\
\hline
\end{tabular}

Table 1: Preconditioning resources required for Arnoldi methods

degrees of freedom and accounts for 20 different material groups. The speed is assumed to be $60 \mathrm{~km} / \mathrm{h}$.

Our aim is to determine approximations to the smallest 180 eigenvalues with relative error smaller than $1 \%$ and the corresponding eigenvectors.

Linearizing problem (1.1) in the usual way

$$
\left(\begin{array}{cc}
-G & -K \\
I & O
\end{array}\right)\left(\begin{array}{c}
\omega x \\
x
\end{array}\right)=\omega\left(\begin{array}{cc}
M & O \\
O & I
\end{array}\right)\left(\begin{array}{c}
\omega x \\
x
\end{array}\right)
$$

or by the Hermitian problem

$$
\left(\begin{array}{cc}
i G & K \\
K & O
\end{array}\right)\left(\begin{array}{c}
\lambda x \\
x
\end{array}\right)=\lambda\left(\begin{array}{cc}
M & O \\
O & K
\end{array}\right)\left(\begin{array}{c}
\lambda x \\
x
\end{array}\right)
$$

and applying the shift-and-invert Arnoldi method requires an LU factorization of $Q(\omega)$ or $\tilde{Q}(\lambda)$ for every shift. Since the eigenvalues of (4.1) are purely imaginary and a large number of eigenvalues distributed in a large interval is wanted, the shifts $\omega$ have to be chosen imaginary as well, and $Q(\omega)$ is a complex matrix. Determining the factorization by SuperLU [5] requires a memory of 6.04 GByte and a CPU time of 3910 seconds on one PA-RISC (750 MHz) processor of an HP superdome.

Applying the nonlinear Arnoldi method [26] the preconditioners can be chosen as real matrices $K-\omega^{2} M$, the LU factorization of which requires 2.7 GByte storage and 1940 seconds with SuperLU, and 2.86 GByte storage and 1080 seconds with the multi frontal solver MA57 of HSL [10]. Since the LU factorization has to be updated several times a total CPU time of more than 12 hours results on one processor of the superdome. Table 1 summarizes the resources required for preconditioning the linear and nonlinear Arnoldi methods.

AMLS demands much less storage and the problem under consideration can be solved on a personal computer, namely a Pentium 4 processor with $3.0 \mathrm{GHz}$ and 1 GByte storage. With a cut-off frequency of $\omega_{c}=5 \times 10^{9}$ the problem is projected to a gyroscopic eigenproblem (3.2) of dimension $n_{c}=2635$ requiring a CPU time of 976 seconds under MATLAB 7.0. Solving the linearization (3.3) of the projected problem (3.2) by eigs (i.e. by ARPACK) requires another 124 seconds. $0.65 \%$.

Fig. 3 shows the relative errors of all 180 eigenvalues which are all smaller than

The solution time of the projected problem (3.2) can be further reduced projecting it to the 262 dimensional subspace spanned by the eigenvectors of the linear problem (3.4) corresponding to eigenvalues smaller than $1.5 \omega_{\max }^{2}$ and solving the projected problem by the dense solver eig. This way the computing cost is decreased to 35.9 seconds to solve (3.4), 3.5 seconds to obtain the projected problem, and 7.3 seconds to solve it. The maximum relative error is raised only to $0.68 \%$

Table 2 summarizes the timings for the AMLS reduction and the solution of the reduced eigenproblem by the implicitly restarted Arnoldi method (ARPACK) and 


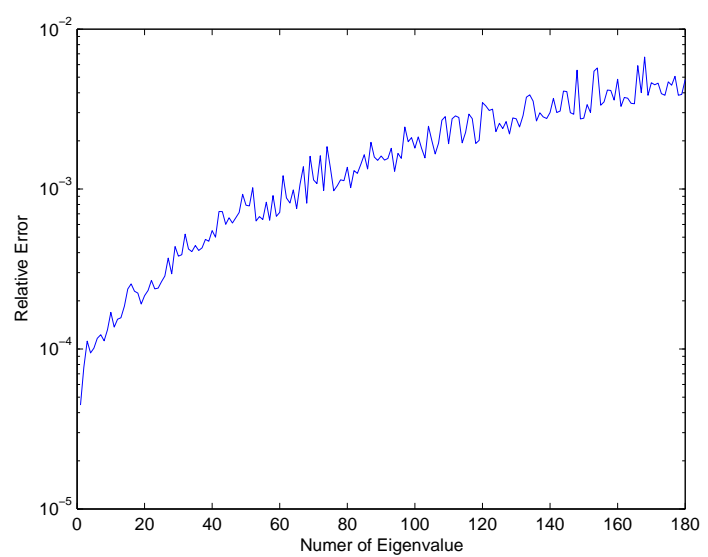

Fig. 3: Relative errors of smallest 180 eigenvalues

\begin{tabular}{|r|r|r|r|r|r|r|}
\hline cut-off freq. & $\omega_{c}$ & red.time & ARPACK & rel. error. & projection & rel. error \\
\hline $5 \times 10^{9}$ & 2635 & 976 sec. & 124 sec. & $0.65 \%$ & 47 sec. & $0.68 \%$ \\
$1 \times 10^{10}$ & 4194 & 984 sec. & 255 sec. & $0.28 \%$ & 133 sec. & $0.32 \%$ \\
$2 \times 10^{10}$ & 6898 & 1067 sec. & 547 sec. & $0.14 \%$ & 289 sec. & $0.18 \%$ \\
\hline
\end{tabular}

Table 2: CPU timing for AMLS reduction and solution

by projecting it to an invariant subspace of the linear part and subsequent solution of the projected problem by a dense solver for different cut-off frequencies $\omega_{c}$. It demonstrates that the time for reducing the problem by AMLS does not depent very sensitively on $\omega_{c}$, but increasing the cut-off frequency results in substantial growth of the time for solving the projected problem.

Acknowledgements. The first author gratefully acknowledges financial support of this project by the German Foundation of Research (DFG) within the Graduiertenkolleg "Meerestechnische Konstruktionen". Thanks are due to Udo Nackenhorst who provided us with the finite element model of the belted tire.

\section{REFERENCES}

[1] W.E. Arnoldi. The principle of minimized iterations in the solution of the matrix eigenvalue problem. Quart. Appl. Math., 9:17-29, 1951.

[2] J.K. Bennighof and M.F. Kaplan. Frequency sweep analysis using multi-level substructuring, global modes and iteration. In Proceedings of the AIAA 39th SDM Conference, Long Beach, Ca., 1998.

[3] J.K. Bennighof and R.B. Lehoucq. An automated multilevel substructuring method for the eigenspace computation in linear elastodynamics. SIAM J. Sci. Comput., 25:2084-2106, 2004.

[4] T. Betcke and H. Voss. A Jacobi-Davidson-type projection method for nonlinear eigenvalue problems. Future Generation Computer Systems, 20(3):363 - 372, 2004.

[5] J.W. Demmel, J.R. Gilbert, and X.S. Li. SuperLU Users' Guide. Technical Report LBNL-44289, Lawrence Berkeley National Laboratory, 2003. Available at http://crd.Ibl.gov/ xiaoye/SuperLU/.

[6] R.J. Duffin. The Rayleigh-Ritz method for dissipative and gyroscopic systems. Quart. Appl. Math., 18:215 - 221, 1960.

[7] K. Elssel and H. Voss. An a priori bound for automated multilevel sub- 
structuring. Technical Report 81, Institute of Mathematics, Hamburg University of Technology, 2004. To appear in SIAM J.Matr.Anal.Appl. Available at http://www.tu-harburg.de/mat/Schriften/rep/rep81.pdf.

[8] K. Elssel and H. Voss. A modal approach for the gyroscopic quadratic eigenvalue problem. In P. Neittaanmäki, T. Rossi, S. Korotov, E. Onate, J. Periaux, and D. Knörzer, editors, Proceedings of the European Congress on Computational Methods in Applied Sciences and Engineering. ECCOMAS 2004, Jyväskylä, Finland, 2004. ISBN 951-39-1869-6. Available at http://www.tu-harburg.de/mat/Schriften/rep/rep73.pdf.

[9] B. Hendrickson and R. Leland. The Chaco User's Guide: Version 2.0. Technical Report SAND94-2692, Sandia National Laboratories, Albuquerque, 1994.

[10] HSL2002: A catalogue of subroutines, 2002. Available at http://www. aspentech.com/hsl/cat_hsl2002.pdf.

[11] M.F. Kaplan. Implementation of Automated Multilevel Substructuring for Frequency Response Analysis of Structures. PhD thesis, Dept. of Aerospace Engineering \& Engineering Mechanics, University of Texas at Austin, 2001.

[12] G. Karypis and V. Kumar. Metis. a software package for partitioning unstructured graphs, partitioning meshes, and computing fill-reducing orderings of sparse matrices. version 4.0. Technical report, University of Minnesota, Minneapolis, 1998.

[13] A. Kropp and D. Heiserer. Efficient broadband vibro-acoustic analysis of passenger car bodies using an FE-based component mode synthesis approach. J.Comput.Acoustics, 11:139 157,2003

[14] C. Lanczos. An iteration method for the solution of the eigenvalue problem of linear differential and integral operators. J. Res. Nat. Bur. Standards, 45:255-282, 1950.

[15] R.B. Lehoucq, D.C. Sorensen, and C. Yang. ARPACK Users' Guide. Solution of Large-Scale Eigenvalue Problems with Implicitly Restarted Arnoldi Methods. SIAM, Philadelphia, 1998.

[16] K. Meerbergen. Locking and restarting quadratic eigenvalue solvers. SIAM J. Sci. Comput., $22: 1814-1839,2001$.

[17] V. Mehrmann and H. Voss. Nonlinear eigenvalue problems: A challenge for modern eigenvalue methods. GAMM Mitteilungen, 27:121 - 152, 2004.

[18] V. Mehrmann and D. Watkins. Structure-preserving methods for computing eigenpairs of large sparse skew-Hamiltonian/Hamiltonian pencils. SIAM J. Sci. Comput., 22:1905 - 1925, 2001.

[19] U. Nackenhorst. Rollkontaktdynamik - Numerische Analyse der Dynamik rollender Körper mit der Finite Elemente Methode. Habilitationsschrift, Institut für Mechanik, Universität der Bundeswehr, Hamburg, 2000.

[20] U. Nackenhorst. The ALE-formulation of bodies in rolling contact. Theoretical foundations and finite element approach. Comput. Meth. Appl. Mech. Engrg., 193:4299-4322, 2004.

[21] U. Nackenhorst and O. von Estorff. Numerical analysis of tire noise radiation - a state of the art review. In Inter-noise 2001. The 2001 International Congress and Exhibition on Noise Control Engineering, The Hague, The Netherlands, 2001.

[22] A. Ruhe. Rational Krylov, a practical algorithm for large sparse nonsymmetric matrix pencils. SIAM J. Sci. Comp., 19(5):1535-1551, 1998.

[23] G.L. Sleijpen and H.A. van der Vorst. A Jacobi-Davidson iteration method for linear eigenvalue problems. SIAM J.Matr.Anal.Appl., 17:401 - 425, 1996.

[24] R. Stryczek, A. Kropp, S. Wegner, and F. Ihlenburg. Vibro-acoustic computations in the midfrequency range: efficiency,evaluation, and validation. In Proceedings of the International Conference on Noise $\&$ Vibration Engineering, ISMA 2004, KU Leuven, 2004. CD-ROM ISBN 90-73802-82-2.

[25] F. Tisseur and K. Meerbergen. The quadratic eigenvalue problem. SIAM Review, 43:235 - 286, 2001.

[26] H. Voss. An Arnoldi method for nonlinear symmetric eigenvalue problems. In Online Proceedings of the SIAM Conference on Applied Linear Algebra, Williamsburg., http://www.siam.org/meetings/laa03/, 2003.

[27] C. Yang, W. Gao, Z. Bai, X. Li, L. Lee, P. Husbands, and E. Ng. An algebraic sub-structuring method for large-scale eigenvalue calculations. SIAM J. Sci. Comput., 27:873-892, 2005. 